\title{
Image - discours et discours sur l'image
}

\section{Guy Dufau}

\section{OpenEdition}

\section{Journals}

Édition électronique

URL : http://journals.openedition.org/trema/2415

DOI : 10.4000/trema.2415

ISSN : 2107-0997

\section{Éditeur}

Faculté d'Éducation de l'université de Montpellier

\section{Édition imprimée}

Date de publication : 1 décembre 1992

Pagination : 83-90

ISSN : 1167-315X

\section{Référence électronique}

Guy Dufau, «Image - discours et discours sur l'image », Tréma [En ligne], 2| 1992, mis en ligne le 01 décembre 1992, consulté le 19 avril 2019. URL : http://journals.openedition.org/trema/2415 ; DOI : 10.4000/trema.2415

Ce document a été généré automatiquement le 19 avril 2019

Trema 


\title{
Image - discours et discours sur l'image
}

\author{
Guy Dufau
}

1 Dans les années soixante on nous parlait beaucoup du "professeur et des images ». Davantage du professeur, du reste, que des images. L'image déferlait sur nous, la télévision se démocratisait et nous nous demandions ce que l'on allait pouvoir en faire, avec une double inquiétude ainsi résumée :

- si nous n'introduisons pas ces moyens « modernes » dans notre enseignement, on va nous prendre pour des enseignants d'un autre âge ;

- si nous plaçons des circuits fermés partout, nous allons devenir des professeurs inutiles, tout juste bons à gérer le « feed-back».

2 On nous parlait aussi, parallèlement, d'une science des signes qui allait englober les sciences du langage, c'était annoncé. L'on nous donnait à penser que l'image, sans qu'on puisse encore bien repérer la double articulation - mais ça viendrait - fonctionnait comme un langage. Nous nous lancions alors dans une réflexion sur l'image, où se croisaient les choses savantes que nous avions lues et les choses plus modestes qui s'attachaient à notre existence. Je me demandais, notamment, pourquoi, ayant été placé, encore étudiant, devant la «Rendición de Breda » familièrement appelée les «Lanzas », de D. Diego Velazquez de Silva, familièrement appelé Velazquez, j'avais entendu, je dis bien: entendu, la phrase du roi : "Marqués de Spinola, tomad a Breda " (Marquis de Spinola, prenez Breda). Pedro de Madrazo, auteur du catalogue de 1907 du musée du Prado a entendu, quant à lui, en admirant « las Lanzas ", un dialogue entre Spinola et Justino de Nassau. Nassau, "en posture déférente ", remet les clefs de la ville et remercie son vainqueur d'avoir respecté scrupuleusement les lois de la guerre. Spinola, en face, vainqueur et généreux, complimente Nassau pour la vaillance qu'il a toujours montrée. C'est de ce croisement, de cette hybridation, que je souhaite rendre compte aujourd'hui. Les titres des paragraphes renvoient à des lectures savantes, oubliées pour la plupart, mais qui ont sédimenté pendant plus d'un quart de siècle aujourd'hui. Le reste, il faut le signaler, n'est pas nécessairement autobiographique. 


\section{1 - «Seul tu n'es rien, il faut que quelqu'un te nomme »}

3 L'image, comme bien d'autres choses qui n'ont rien à voir avec elle, pourrait bien n'avoir d'existence que dans le discours. Oui, bien sûr, le papier est là ; je peux le toucher et en apprécier le grain; et si je soumettais cette plaque rectangulaire de papier photographique, et toutes celles qui lui sont semblables, à l'analyse, j'y découvrirais un fonds commun fait de traces d'argent, de restes d'iodure, de gélatinobromure, d'oxalates et que sais-je d'autre encore, qui lui conférent une réalité concrète. L'image est bien là ; elle a une réalité. On dit même qu'elle a un statut ontologique, lié au fait qu'elle transforme les apparences en réalité. Comme tout objet, pourtant, elle ne dit d'abord que ce qu'elle sait sur soi, qu'une chose, et c'est qu'elle est (es ist). Mais elle le dit. Toutefois, un objet n'en dit pas autant que le français le suggère, «parce que s'il disait qu'il est, par là même il se nommerait «il ». Il dit seulement: "es ist », «il y a quelque chose là ", «prenez-le en considération ». La formule de Hegel est neutre et reste aussi indéterminée qu'elle le peut $»$.

4 Je sais donc de l'image que c'est une image, une apparence ou une représentation, parce qu'on me l'a dit et parce que j'en parle, parce que j'y suis, en un mot, préparé.

5 Avant que l'on m'en parle, j'étais, à peu de choses près, dans la situation du Papou qui découvre pour la première fois sa propre image dans un miroir. Ou bien, peut-être, dans la situation de mon chien, lequel, découvrant son image dans un grand miroir, passe derrière celui-ci pour aller à la rencontre de l'intrus. Ce même chien, du reste, ne sait pas voir les chiens à la télévision. Il ne s'inquiète que lorsque Lassie ou Rintintin ou quelques autres, sur l'écran, se mettent à aboyer. Alors, infailliblement, il se dirige vers le hautparleur. Supériorité de l'oreille sur l'œil ? « Eh bien vous l'avez vue, et moi je l'ai sentie.» En espagnol on « sent » les bruits : « me parece que he sentido un ruido » vous déclare-ton parfois. La Fontaine, en plaçant le vers cité plus haut dans la bouche de l'un des deux plaideurs se disputant une huître, affirmait-il cette prééminence de l'oreille? Après tout, on aime bien écouter les coquillages. Ils nous rappellent le bruit des vagues. Ils nous parlent de la mer.

6 L'image pourrait bien être le résultat d'une analyse faite avec des mots. Le sens, en effet, n'adhère pas à l'image mais au sensible qu'elle est censé représenter. Censé est bien le mot, car l'image a transformé l'être humain ou les autres choses qu'elle représente en spectacle. Elle recherche un effet, et fait de l'effet. Elle représente les choses telles qu'on ne les verra jamais. Elle les déguise et les travestit. Le cinéma notamment regarde les acteurs sous le nez et sous le nombril parfois, comme on n'osera jamais le faire avec un œil ordinaire. CEil voyeur de la caméra et vertige du gros plan; ici tout le monde avance masqué. Je me souviens aussi des efforts désespérés d'un instituteur qui apprenait le français à des « rejoignant », comme on dit aujourd'hui (on les avait appelés, d'abord, les primo-arrivants mais cela faisait un peu maladie honteuse). Il y avait eu pour la génération de la guerre la primo-infection et il y avait aussi les discours « faschoïdes » du moment. Tout ceci se croisant, on en vint à fabriquer un autre mot dont l'élégance est douteuse, à n'en pas douter, mais dont l'existence même tendrait à prouver que, par le mot, on espère agir sur la perception des choses: «la noire à faire peur une brune adorable » Molière- « Le Misanthrope »). Cet instituteur, donc, utilisait des images comme support de son enseignement. L'image signifiant universel, croyait-il, l'aiderait à se faire comprendre. Il en avait une, notamment, qui représentait un chat, perché sur une table, 
buvant le lait contenu dans une assiette. La phrase qui accompagnait l'image était la suivante : «Le chat boit le lait ». L'incompréhension régnait. Il n'y a ni chat, ni tables, ni lait qui traîne dans le pays d'où venaient ces enfants. Cette image n'avait pas de sens puisque le sens est dans l'expérience, dans le sensible, ou, à défaut, dans les mots qui renvoient, au bout du compte, au terme d'une longue anastomose, à l'expérience. Et, personne dans l'assistance n'ayant eu l'expérience du chat buvant le lait, personne ne sut ce que le discours sur le chat voulait dire et l'image, seule, n'est jamais parvenue à créer du sens.

7 De la même manière, mon professeur d'anglais, moderne et audio-visuel, parlait sur des images. Il y avait celle du petit déjeuner avec son petit pot de "strawberry jam ». Une fraise était dessinée sur l'étiquette. Mais, dans ce continuum sonore : «straw..etc » que l'on parvenait, peut-être, à découper au moyen des toniques, quelle était la partie qui signifiait «fraise » et quelle était celle qui signifiait, quoi au juste ? confiture? gelée? compote? Qui a dit signifiant universel?

8 Je n'ai jamais connu mon grand père, Pierre Rouquette ; ma mère non plus. « Qui est cet homme en uniforme? » lui ai-je un jour demandé alors que je jouais avec la photo de mon grand père qui n'était plus, et allait soudain comme par miracle reprendre une sorte de vie.

9 - « C'est ton grand père, mon père, mort en Belgique en 14. La guerre n'a pas beaucoup duré pour lui.»

Il était important à ses yeux de me narrer la Grande Guerre. Ses Institutrices avaient lu Déroulède :

\section{"Oh femme ta tendresse a déformé cette âme.}

S'il ne sait pas mourir, tu ne sais pas aimer. »

11 Il lui paraissait nécessaire de me parler avec conviction du sacrifice suprême. Et la voilà partie dans une longue histoire qu'elle n'avait pas vécue, qu'elle tenait de sa mère et qu'elle se remémorait à chaque fois qu'elle passait en revue les photos de famille rangées dans un carton à chaussures. Cette revue s'effectuait périodiquement, à la suite d'une noce ou d'un baptême, dont la représentation, (le souvenir), sous forme d'image, allait grossir la collection des représentations, conservée dans la boîte à chaussures. J'ai quitté très tôt la maison de ma mère. La collection s'est enrichie pendant mon absence. Mes cousins ont eu des enfants que je ne connais pas et qui ont eu, à leur tour, des enfants qui ne me sont rien. J'ai récupéré la collection à la mort de ma mère. Elle n'était plus là pour me parler de ses chers bouts de carton. Ils ne me disent rien. J'ai perdu ma mémoire. Seule me parle encore la photo de mon grand père ; elle me parle avec la voix de ma mère, avec la prosodie qui était sienne et avec ses mots.

A l'autre bout de la chaîne, et comme une confirmation de ce que je sens, il y a une petite fille de six ans qui me reproche sans cesse de faire des photos qui n'en sont pas. Elle ne supporte pas les natures mortes, les commodes ou les sofas. «Ce ne sont pas des photos, dit-elle, on n'a rien à en dire ». Elle ne sait pas encore parler des objets inanimés, de la patine du bois ou de ses superbes reflets. Une photo, selon ce qu'elle affirme, ne peut que représenter quelqu'un dont on peut dire qui il est et comment on le trouve : «Tu aurais dû te raser sur cette image. Tu aurais l'air moins rébarbatif. Maman est très bien sur ce cliché » etc... Elle me dit, à sa manière, qu'une photo dont on ne peut parler n'existe pas. Ce qui revient encore à dire que c'est le discours qui confère une existence à l'image. Le 
discours du physicien et du chimiste qui s'est trouvé placé au tout commencement, puis le discours de celui qui aime ou se souvient et qui assure au cliché une sorte de pérennité.

Nota bene qui n'a rien à voir : Les êtres intersubjectifs, les réalités qui apparaissent entre deux ou plusieurs sujets parce qu'ils en parlent, auraient-ils besoin de voir leur existence confirmée par l'image? Il y a ceux qui multiplient les images de Dieu, par exemple, et ceux qui veulent à tout prix, posséder un Saint Suaire gardant l'empreinte de la Sainte Face. Il faut admettre qu'il y a aussi ceux qui cassent les statues et brûlent les images. Veulent-ils, ceux-ci, affirmer la primauté du verbe?

\section{2 - « Analogon de l'objet absent »}

14 J'ai sous les yeux la photo de Marie. Je me dis que c'est Marie et je finis, tant mon discours est convaincant, par croire que c'est Marie, en effet. Je ne pourrais « voir » Marie dans ce bout de papier si je ne me laissais abuser par mon propre discours, sous-tendu par ma propre émotion. Si je ne laissais, comme on dit si bien : «parler mon cœur ». Et le cœur parle et il parle fort. Et je suis à ce point abusé par le discours intérieur que je me tiens, que je finis par embrasser la photo de Marie ... un élan que je ne puis maîtriser. C'est alors que je vois bien que ce n'est pas Marie; aucune douceur, aucune chaleur, aucune réaction de femme. C'est alors que la photo retrouve son statut d'analogon. C'est quelque chose qui ressemblait à Marie, mais qui n'était Marie que par un puissant effort de mon imaginaire. Il fallait que je néantise et que je recrée comme disent les philosophes. L'esprit, dans ce cas, est plus occupé qu'il ne l'est avec les succédanés. On pouvait pendant la guerre griller de l'orge comme s'il s'était agi de café. Avec la photo de Marie c'est plus simple et plus subtil à la fois. C'est plus simple car il n'est pas nécessaire d'avoir une adresse, un tour de main ; mais c'est plus compliqué car il faut, par la magie de son verbe intérieur, endormir sa propre raison. Aussi, quelque temps après qu'eut pris fin la belle passion qui me liait à Marie, ai-je brûlé dans ma cheminée ce qui me restait d'elle: un ruban, des lettres, des photos, des cheveux. Tous ces pieux objets « me parlaient » et mon cœur se fendait quand il lui arrivait de les redécouvrir. Mais voyez donc Merleau Ponty et Sartre pour le reste. Et si l'on revient à ce qui était dit plus haut. tant que l'on se souviendra de Pierre, la photo de Pierre parviendra à émouvoir. Lorsque Pierre sera perdu dans les mémoires, la photo ne fera plus vibrer personne.

On tiendra sur elle un autre discours : technique, historique, esthétique... qui la fera exister encore. On parlera contraste ; on parlera sépia ; on dira encore que les militaires, à cette époque là, n'étaient point épargnés par leur coiffeur ni leur tailleur. Mais plus personne, placé devant cette image de Pierre, n'entendra comme l'entendait ma mère, siffler les balles de Mauser qui avaient, sur une morne plaine de Belgique, ôté la vie de Pierre. Est-ce ce qu'il faut comprendre dans le propos du directeur d'une revue récente qui déclarait : « La modernité, c'est un texte sur une image »?

Le dernier coup m'a été porté par l'image de synthèse. Cette image n'est l'image de rien et n'a de réalité que dans le langage. Les deux langages pourrait-on dire. Celui qui porte sur les mots que je dis et qui permet de la concevoir; puis le langage de programmation qui permet d'installer cette image sur l'écran de mon ordinateur, ou dans les supports de sa mémoire, où j'irai la rechercher à chaque fois que je lancerai le programme.

Au tout premier échelon, tout élève de CM2 sait fabriquer en Logo, un octogone, ou une rosace faite d'octogones emboîtés. Il faut le voir « parler » à la machine : 
etc..

- «Que fais-tu là enfant ?»

- " Je dis à la tortue de me faire un octogone. Et puis après, chaque fois que je dirai octogone à l'ordinateur, j'aurai le même dessin. »

- «N'est-ce pas prodigieux?»

- «Non. Si on explique bien ça marche. Mais il faut bien tout lui dire.»

19 L'image de synthèse est au-delà du dessin en logo de l'enfant. Sa création est nettement plus complexe. C'est en fait un espace image; on ne fait plus voir mais interagir. Et les spécialistes de vous parler d'espace virtuel, de «l'être-chose» et de la caméra virtuelle qui permet de le visualiser. Cette caméra peut aller jusqu'à lui, se retourner et donc nous montrer ce qu'il (l'être chose) pourrait voir dans cet espace qui semble exister. Escher avait tout à fait compris cela ("Un autre monde», 1947 gravure sur bois en 3 couleurs. 317X260). Il explique dans cette œuvre, à l'aide d'un oiseau « reconnaissable dans une architecture, que l'on peut donner trois fonctions spatiales à un plan ». («Le monde de Escher ", Eds du Chêne, 1972)

\section{3 - « Le punctum et le studium »}

"On dirait une carte postale ». Voilà ce que me disait quelqu'un en me parlant de la photo dont j'étais fier. Il avait une perception immédiate de l'objet. Il avait été frappé par une forme, des couleurs, un format ou un certain éclat. La carte postale ça saute aux yeux; c'est réaliste. J'étais plutôt vexé. Cette étape de la perception grossière est pourtant nécessaire. Il faut être accroché, comme on dit aujourd'hui ; il faut que quelque chose m'empoigne, avant que je commence à me pencher sur la réalité représentée ... sur ce que l'image est vraiment. Admettons donc que nous ne pouvons parler de l'image que si quelque chose, chez elle, nous invite à le faire. Un je ne sais quoi qui me "point », ainsi que le dit Barthes; qui me saisit. Il vaut mieux sans doute ne point trop approfondir la nature de ce "punctum». J'ai repéré, soudain, une photo de la Roumanie dans ma collection. Quelque chose m'a renseigné tout de suite. Comme pour Bourrel dans les Cinq dernières minutes: "Mais c'est bien sûr... ». C'est une donnée immédiate ; légèrement antérieure au discours. Juste avant qu'il ne vienne investir l'image de manière à m'aider à l'étudier plus finement et, ainsi, à légitimer l'opinion soudaine que j'avais eue.

21 Mais oui, bien sûr, c'est le brancard bizarre de cette charrette typique, ce sont ces nénuphars énormes et ce lac bleu qui ont réactivé la Roumanie. Laissons régner le flou autour de ces impressions; essayons de faire en sorte que le discours ne vienne pas investir ce territoire là, sans cela, le je ne sais quoi, le détail, le presque rien qui m'attirait vers la photo de Pierre sera submergé par le verbe et il n'y aura plus aucun interstice, aucun jeu, entre l'image et le discours. Ou alors, démesure plus grande encore, l'image sous-tendra un autre type de discours. Un discours ample, métaphysique quasiment, sur l'aptitude de l'homme à représenter, c'est à dire à créer.

22 Un discours qui pourrait faire fond sur les expériences d'une vie et dont les points saillants, par exemple, pourraient être les suivants :

- Tout jeune, j'apprends, à l'école, que la Corse est une île. 
- Plus tard, j'apprends à la reconnaître dans ses représentations : carte ou modèle qui a sans doute inspiré le bijou abstrait que les Corses portent à leur cou.

- Plus tard encore, je vais en Corse et je ne vois rien. J'y rencontre, dans le Centre profond, des bergers qui n'ont jamais vu la mer. Les affixes péjoratifs (Ghisoni><Ghisonaccia) montrent en quelle estime ces insulaires tiennent la mer et les lieux qui l'avoisinent.

- Il y a quelques années, enfin, je vois une photo de la Corse par satellite et je la reconnais. Je me demande comment c'est possible. Je me dis que le logos ce n'est tout de même pas n'importe quoi. Je finis même par me dire qu'enseigner c'est faire partager ces moments d'intense jubilation qui découlent de la prise de conscience de ce que l'intelligence ce n'est pas le propre des singes.

Ils faut qu'ils sachent, nos petiots, relativiser quand on leur parle du langage des abeilles, de celui des cervidés ou bien, encore, de celui des chimpanzés nourris au sein. Et j'espère bien qu'il est plein de remords, quand il voit la photo de la terre éditée par la NASA, cet élève arrogant qui m'avait dit, alors que je lui parlais de la Terre «bleue comme une orange ", qu'il avait rarement entendu pareille absurdité. Le poète avait vu juste. Il suffit aujourd'hui de regarder. Mais j'y songe, qu'avait-il regardé, lui, le poète ?

\section{BIBLIOGRAPHIE}

1 - BENDA Julien : Lettres à Melisande pour son éducation philosophique, Le Livre 1926

2 - TARDY Michel : Le professeur et les images. PUF sup 1966

3 - D. Pedro de MADRAZO : Catalogo de los cuadros del museo nacional depintura y escultura. Imprenta y Fototipia de J. Lacoste Madrid 1907

4 - PARAIN Brice : Recherche sur la nature et les fonctions du langage. NRF 1942

5 - M.O.T.S N³1 Mars 92 Presses de la Fondation Nationale des Sciences Politiques

6 - MERLEAU PONTY Maurice : Signes. NRF 1960 - Phénoménologie de la perception. NRF 19456 SARTRE Jean-Paul : L'imaginaire. NRF idées 1940

7 - BARTHES Roland : La chambre claire : Notes sur la photographie Gallimard Le seuil 1980.

\section{AUTEUR}

\section{GUY DUFAU}

IEN, IUFM de Montpellier 